\title{
Scholarly

\section{Severe Invasive Group A Streptococcal Disease with Rhabdomyolysis but without Evidence of Shock or Local Myositis}

\author{
Arthur Okere, ${ }^{1}$ David Schwartz, ${ }^{1}$ Philip Tierno, ${ }^{2}$ Patrick Schlievert, ${ }^{3}$ and Randa Hamadeh ${ }^{1}$ \\ ${ }^{1}$ Department of Medicine, New York University Medical Center, New York, NY 10016, USA \\ ${ }^{2}$ Laboratory Medicine, New York University Medical Center, New York, NY 10016, USA \\ ${ }^{3}$ Department of Microbiology, University of Minnesota, Minneapolis, MN 55812, USA
}

Correspondence should be addressed to Arthur Okere, arthur.okere@nyumc.org

Received 11 February 2009; Accepted 12 March 2009

We report a presentation in an adult with Streptococcus pyogenes bacteremia and invasive tissue disease with rhabdomyolysis, but without evidence of toxic shock syndrome or local myonecrosis. We thus investigated how this infecting strain differed from strains known to cause toxic shock syndrome.

Copyright (c) 2009 Arthur Okere et al. This is an open access article distributed under the Creative Commons Attribution License, which permits unrestricted use, distribution, and reproduction in any medium, provided the original work is properly cited.

\section{Introduction}

Severe invasive group A streptococcal infections have been previously described to cause bacteremia, necrotizing fasciitis, gangrenous myositis, pneumonia, or other possible syndromes associated with this organism being isolated from a usually sterile area of the body [1]. Group A streptococcal toxic shock syndrome (TSS) has been defined as a clinical syndrome characterized by hypotension (at the time of admission or within four to eight hours), as well as two or more signs of organ failure, such as liver or renal dysfunction, adult respiratory distress syndrome, erythematous macular rash, coagulopathy, or soft tissue necrosis [2]. We report a presentation in an adult with group A streptococcal bacteremia and other manifestations of toxic shock syndrome, however, not found to have the associated shock.

\section{Case Report}

A 41-year-old male was brought to the Emergency Room (ER) after having called 911 with complaints of right ear pain and discharge, decreased hearing acuity, headache, dizziness, and subjective fevers over the 3 days prior to admission. His past medical history is only significant for heavy alcohol abuse, methylenetetrahydrofolate reductase (MTHFR) deficiency with concomitant hyperhomocysteinemia and multiple DVT/PEs. According to family members, he experiences a baseline tremor in the upper extremities of unknown etiology, unassociated with alcohol usage or withdrawal episodes. The patient admits to having a young child who was recently treated for Streptococcus pyogenes pharyngitis. His only medication is daily Coumadin for his hypercoagulable state. He denies alcohol consumption in the past week as well as any usage of IV drugs.

When seen in the ER, he was observed to be alert and oriented, tremulous, febrile to $103^{\circ} \mathrm{F}$, tachycardic and normotensive. Physical exam was only notable for serous discharge from the right ear, warmth, erythema, and swelling of the right forearm, elbows and knees, bilaterally. The examinations of his heart, lungs, and abdomen were unremarkable; neurological exam was nonfocal. Laboratory tests initially revealed serum creatinine of $1.2 \mathrm{mg} / \mathrm{dL}$ and transaminases of AST/ALT 152/65 U/L, total bilirubin $1.6 \mathrm{mg} / \mathrm{dL}$, conjugated $0.3, \mathrm{Hgb} 14.6 \mathrm{~g} / \mathrm{dL}$, HCT $41.2 \%$, platelet count $118 \times 10^{9} / \mathrm{L}$, albumin $3.7 \mathrm{~g} / \mathrm{dL}$. Initial urinalysis findings were a specific gravity 1.028, $\mathrm{pH} 5.5$, small bilirubin, trace ketones, protein $>299$, positive nitrites, small leukocyte esterase, large blood, with 6-10 red blood cells, 3-10 white blood cells, urine cultures with no growth.

Laboratory tests 8 hours later revealed serum creatinine and transaminases of $1.6 \mathrm{mg} / \mathrm{dL}$ and AST/ALT 1867/573 U/L respectively, as well as rhabdomyolysis with serum creatine phosphokinase of $8030 \mathrm{U} / \mathrm{L}$. His serum alcohol, serum and urine toxicology screen were all negative. The patient, who had initially been administered valium in the ER for presumed alcohol withdrawal, was later found to be somnolent, and in respiratory distress requiring intubation. 
He was admitted to the ICU for further monitoring, broad spectrum antibiotics, and intravenous fluid management. A delayed lumbar puncture was performed a few hours after antibiotics, with CSF revealing total protein $234 \mathrm{mg} / \mathrm{dL}$, glucose $69 \mathrm{mg} / \mathrm{dL}$, nucleated cells of $205 / \mathrm{mm}^{3}$, neutrophils $92 \%$, lymphocytes $7 \%$, monocytes $1 \%$, final CSF gram stain, and cultures being negative.

During his stay in the intensive care unit, he continued to have intermittent fevers. Admission blood cultures were positive for Streptococcus pyogenes, susceptible to penicillin. Blood culture bottles were obtained using the BacT/Alert 3D Blood Culture System, Biomerieux, Inc., Durham, NC, USA. Antibiotic susceptibility was performed with Vitek Two, Biomerieux, Inc., St Louis, Mo, USA. Antibiotics were later narrowed to penicillin $\mathrm{G}$, with clindamycin and 3 doses of IVIG administered in case of toxin production. Serum creatine phosphokinase levels continued to rise daily, with concomitant liver and renal dysfunction. Given the findings of warmth, erythema, and swelling of the elbows and knees bilaterally, joint fluid was obtained from these sites which also grew Streptococcus pyogenes. CT scans of the head was within normal limits, and scans of the body/extremities resulted in no findings of focal myositis. A transthoracic echocardiogram revealed no valvular vegetations, and an ENT exam was significant for otitis media. Lower extremity dopplers were also obtained, which revealed no evidence for acute deep vein thrombosis.

On hospital day 3, the patient remained intubated, however alert, neurologically nonfocal, and responsive to commands. The patient remained intermittently febrile, however hemodynamically stable, with no significant drop in blood pressure since admission. Serum creatine phosphokinase levels peaked at 139,781 U/L on hospital day 4, with subsequent decline. After serum AST/ALT values peaked at $3217 / 1046 \mathrm{U} / \mathrm{L}$ on hospital day 3, and serum creatinine $4.0 \mathrm{mg} / \mathrm{dL}$ on hospital day 6 , hepatic and renal function likewise improved. By hospital day 7, the patient underwent successful extubation, and was fully responsive, afebrile, and hemodynamically stable. His last recorded transaminases were AST/ALT 43/31 U/L. He was discharged home in stable condition on hospital day 10.

Clinical isolates were obtained from cultures of specimens submitted on the patient. Identification of bacteria suspected of being streptococci was identified according to standard bacteriologic criteria. Group A streptococci were initially identified by their susceptibility to bacitracin and then were grouped in the clinical microbiology laboratory using StrepPro Streptococcal Grouping Kit (Hardy Diagnostics, Santa Maria, Calif, USA). The technique employed was latex agglutination. The patient's Streptococcus pyogenes was found to be positive by antibody testing for both streptococcal pyrogenic exotoxin B and C (SPE B \& C); but the SPE A was negative [3].

Group A streptococcus (Streptococcus pyogenes) is an aerobic gram-positive coccus that causes pharyngitis and a spectrum of skin and soft tissue infections such as impetigo, erysipelas, and localized cellulitis [4]. Less commonly, group A streptococci cause invasive diseases such as bacteremia, necrotizing fasciitis, pneumonia and postpartum sepsis, and the infection may be complicated by TSS in approximately one-third of patients. The entry points for streptococcal infections are the pharynx, skin, or vagina. Note, however, that in those subjects diagnosed with streptococcal TSS, a point of entry cannot be clearly identified in 45 percent [1].

Group A streptococcal TSS (infection associated with the acute onset of shock and organ failure), similar to staphylococcal TSS, is mediated by toxins that act as superantigens, which activate the immune system by circumventing the typical antigen-mediated immune response pathway. Superantigens do not require processing by antigen presenting cells but interact directly with relatively invariant regions of the class II MHC molecules and outside the groove area [5]. Superantigens are capable of interacting with an exceedingly large number of $\mathrm{T}$ cells. Unlike a conventional antigen, which evokes a response from less than $0.01 \%$ of the entire T-cell repertoire, a superantigen can interact with $5 \%$ to $30 \%$ of $\mathrm{T}$ cells [6]. This leads to a release of large quantities of inflammatory cytokines, which results in leakage of capillaries and tissue damage, subsequently leading to shock and multiorgan failure $[4,7]$.

It is well known that streptococcal pyrogenic exotoxins have also been described in group A streptococci, including types $A, B$, and $C$, which are genetically distinct from each other. These toxins have been shown to be superantigens, and to cause TSS in animals. In the majority of reported cases of severe group A streptococcal infection, we have found combinations of SPE A, SPE B, or SPE C to be present, with SPE A being most common in the US [2, 8-12]. Reports have shown that the exotoxin SPE B is largely found in the UK and in Sweden [13,14], and there are others characterized such as streptococcal superantigen, which has been found in M3 strains [15], and mitogenic factor (MF), found in various different M-types [16, 17].

Interestingly, most streptococcal TSS isolates make SPE A or at least contain the gene for SPE A (speA), compared with $15 \%$ of non-TSS isolates [5]. All of the streptococcal-TSS isolates also carry the SPE B or contain the gene for that toxin (speB); but this is also true of non-TSS strains [5]. Although recent evidence suggests that SPE C may also contribute to the illness, the role of SPE B is unclear because both TSS and non-TSS isolates carry the make this product $[18,19]$. Therefore, it is likely that our strain may not result in shock; because the SPE A exotoxin is absent.

As our patient had a strain of $S$. pyogenes that did produce both SPE B as well as SPE C which have been shown to be involved with some TSS-like disease, it is possible that our patient had a MHC II haplotype which was partially protective. MHCs are highly polymorphic, and given that there are over 100 different alleles for class I and class II genes in humans, enormous polymorphism exists [18]. Theoretically, $>10^{12}$ is the diversity possible [20]. Susceptibility to a given pathogen or its toxins may reflect the role of particular MHC alleles in responsiveness or nonresponsiveness to antigens.

Of particular significance is the clinical presentation of this patient relating to the diagnosis of streptococcal TSS. The presence of rapidly progressive end organ failure with positive blood cultures often accompanies streptococcal TSS. 
It has, however, been reported that almost 50 percent of patients with TSS are found to be normotensive on presentation; however even these patients later become hypotensive within the subsequent hours $[7,21]$. Interestingly, our patient was never found to be hypotensive. We therefore have a presentation of an adult with disseminated group A streptococcal bacteremia with severe sepsis and nonfocal rhabdomyolysis without associated shock. We believe that our patient presented with these findings as a result of the absence of SPE A as well as a possible partial protective effect of the MHC II haplotype. It is important for clinicians to be aware that Streptococcus pyogenes can present with toxic shock-like syndromes, with sepsis and severe organ dysfunction, without evidence of shock.

\section{References}

[1] E. M. Adams, S. Gudmundsson, D. E. Yocum, R. C. Haselby, W. A. Craig, and W. R. Sundstrom, "Streptococcal myositis," Archives of Internal Medicine, vol. 145, no. 6, pp. 1020-1023, 1985.

[2] K. Belani, P. Schlievert, E. L. Kaplan, and P. Ferrieri, "Association of exotoxin-producing group A streptococci and severe disease in children," Pediatric Infectious Disease Journal, vol. 10, no. 5, pp. 351-354, 1991.

[3] P. Schlievert, personal communication, Department of Microbiology, University of Minnesota.

[4] A. L. Bisno and D. L. Stevens, "Streptococcal infections in skin and soft tissues," The New England Journal of Medicine, vol. 334, no. 4, pp. 240-245, 1996.

[5] P. Schlievert, "Role of superantigens in human disease," Journal of Infectious Diseases, vol. 167, no. 5, pp. 997-1002, 1993.

[6] B. L. Kotzin, "Superantigens and their role in disease," Hospital Practice, vol. 29, no. 11, pp. 59-63, 68-70, 1994.

[7] "Defining the group A streptococcal toxic shock syndrome: rationale and consensus definition. The Working Group on Severe Streptococcal Infections," The Journal of the American Medical Association, vol. 269, no. 3, pp. 390-391, 1993.

[8] A. R. Hauser, D. L. Stevens, E. L. Kaplan, and P. Schlievert, "Molecular analysis of pyrogenic exotoxins from Streptococcus pyogenes isolates associated with toxic shock-like syndrome," Journal of Clinical Microbiology, vol. 29, no. 8, pp. 1562-1567, 1991.

[9] D. R. Johnson, D. L. Stevens, and E. L. Kaplan, "Epidemiologic analysis of group A streptococcal serotypes associated with severe systemic infections, rheumatic fever, or uncomplicated pharyngitis," Journal of Infectious Diseases, vol. 166, no. 2, pp. 374-382, 1992.

[10] J. M. Musser, A. R. Hauser, M. H. Kim, P. Schlievert, K. Nelson, and R. K. Selander, "Streptococcus pyogenes causing toxic-shock-like syndrome and other invasive diseases: clonal diversity and pyrogenic exotoxin expression," Proceedings of the National Academy of Sciences of the United States of America, vol. 88, no. 7, pp. 2668-2672, 1991.

[11] B. Schwartz, R. R. Facklam, and R. F. Breiman, "Changing epidemiology of group A streptococcal infection in the USA," The Lancet, vol. 336, no. 8724, pp. 1167-1171, 1990.

[12] D. L. Stevens, M. H. Tanner, J. Winship, et al., "Reappearance of scarlet fever toxin A among streptococci in the Rocky
Mountain West: severe group A streptococcal infections associated with a toxic shock-like syndrome," The New England Journal of Medicine, vol. 321, pp. 1-7, 1989.

[13] E. T. Gaworzewska and G. Coleman, "Correspondence: group A streptococcal infections and a toxic shock-like syndrome," The New England Journal of Medicine, vol. 321, p. 1546, 1989.

[14] B. Stegmayr, S. Björck, S. Holm, J. Nisell, A. Rydvall, and B. Settergren, "Septic shock induced by group A streptococcal infection: clinical and therapeutic aspects," Scandinavian Journal of Infectious Diseases, vol. 24, no. 5, pp. 589-597, 1992.

[15] J. A. Mollick, G. G. Miller, J. M. Musser, R. G. Cook, D. Grossman, and R. R. Rich, "A novel superantigen isolated from pathogenic strains of Streptococcus pyogenes with aminoterminal homology to staphylococcal enterotoxins $\mathrm{B}$ and C," The Journal of Clinical Investigation, vol. 92, no. 2, pp. 710-719, 1993.

[16] M. Iwasaki, H. Igarashi, Y. Hinuma, and T. Yutsudo, "Cloning, characterization and overexpression of a Streptococcus pyogenes gene encoding a new type of mitogenic factor," FEBS Letters, vol. 331, no. 1-2, pp. 187-192, 1993.

[17] A. Norrby-Teglund, D. Newton, M. Kotb, S. E. Holm, and M. Norgren, "Superantigenic properties of the group A streptococcal exotoxin SpeF (MF)," Infection and Immunity, vol. 62, no. 12, pp. 5227-5233, 1994.

[18] G. A. Bohach, D. J. Fast, R. D. Nelson, and P. Schlievert, "Staphylococcal and streptococcal pyrogenic toxins involved in toxic shock syndrome and related illnesses," Critical Reviews in Microbiology, vol. 17, no. 4, pp. 251-272, 1990.

[19] D. L. Murray, D. H. Ohlendorf, and P. Schlievert, "Staphylococcal and streptococcal superantigens: their role in human disease," ASM News, vol. 61, no. 5, pp. 229-235, 1995.

[20] J. Kuby, Immunology, W. H. Freeman, New York, NY, USA, 2nd edition, 1994.

[21] V. Hríbalová, "Streptococcus pyogenes and the toxic shock syndrome," Annals of Internal Medicine, vol. 108, no. 5, p. 772, 1988. 